\title{
Entwicklung eines Methodenapparates zur Identifikation und Input-/Outputvernetzung von Geschäftsprozessen zur Sicherung eines nachhaltigen Geschäftserfolges in KMU
}

\author{
Ingolf Wohlfahrt
}

\section{Einleitung}

Im Rahmen der Entwicklung und Umsetzung einer Qualitätsstrategie für die Region Berlin-Brandenburg wurden auf Initiative der Qualitätsinitiative Berlin-Brandenburg Schwerpunkte für die Weiterentwicklung des Qualitätsmanagements zur Verbesserung der Wettbewerbsfähigkeit der Unternehmen in der Region abgeleitet. Die Technische Fachhochschule Wildau, selbst Partner der Qualitätsinitiative Berlin-Brandenburg, reichte ein entsprechendes Forschungsprojekt zur Bearbeitung ein, welches durch den Auftraggeber, die RKW Brandenburg GmbH, ebenfalls Partner der Qualitätsinitiative Berlin-Brandenburg, bestätigt wurde. Die Bearbeitung des Forschungsprojektes mit dem Titel „Entwicklung eines Methodenapparates zur Identifikation und Input-/ Outputvernetzung von Geschäftsprozessen als Basis der Sicherung eines nachhaltigen Geschäftserfolges in KMU“ erfolgte in den Jahren 2002 und 2003 durch die Projektgruppe Qualitätsmanagement an der Technischen Fachhochschule Wildau. Im Februar 2003 konnten die Ergebnisse des Forschungsprojektes den Partnern der Qualitätsinitiative Berlin-Brandenburg vorgestellt werden. [1] Dieses Forschungsprojekt wurde durch die Europäische Union und das Land Brandenburg gefördert.

\section{Situationsbeschreibung}

Die primäre Zielstellung eines jeden Unternehmens ist seit jeher ein dauerhafter Geschäftserfolg. Unternehmerische Tätigkeit wird unter den heutigen Bedingungen des herrschenden Verdrängungswettbewerbs und der Ausprägung eines Käufermarktes wesentlich geprägt durch:

- ein hohes Entwicklungstempo technologischer Innovation,

- sich ständig ändernde Arbeitsprozesse sowie

- einen häufigen Wechsel im wirtschaftlichen, sozialen und kundenbezogenen Umfeld.

Der sich so verschärfende Wettbewerb und die Internationalisierung der Märkte erfordern einen Paradigmenwechsel bei der Gestaltung des betrieblichen Qualitätsmanagements als Wettbewerbselement und Management-Tool. Gefragt sind betriebliche Lösungen, welche die Identifikation aktueller und zukünftiger Forderungen interessierter Parteien des Unternehmens in den Mittelpunkt betrieblicher Aktivitäten stellen und deren angemessene Erfuillung auf wirksame und effiziente Weise einen nachhaltigen und dauerhaften Ge- schäftserfolg gewährleisten. In diesen Zusammenhang ist die Weiterentwicklung von Normen und Modellen zum Qualitätsmanagement, wie die Normenfamilie ISO 9000: 2000, das EFQM-Modell für Excellence und andere Regelwerke, einzuordnen. Sie bieten ein breites Spektrum von Forderungen und gedanklichen Ansatzpunkten für die Realisierung des o. g. Paradigmenwechsels. Was sie nicht bieten können und wollen, sind die Methoden zu seiner betriebsspezifischen Umsetzung. Fakt ist aber, dass das Streben nach Excellence eine systematische Arbeit auf der Basis betriebsspezifisch adaptierter Methoden und Werkzeuge des Qualitätsmanagements erfordert. Analysen des Forschungsauftragnehmers zeigen, dass die Realisierung o. g. Forderungen bei der Einrichtung und Gestaltung von QM-Systemen, bei der Weiterentwicklung solcher Systeme sowie bei der Realisierung von TQMKonzepten in kleinen und mittleren Unternehmen (KMU) eine sehr anspruchsvolle Aufgabenstellung darstellt, auch weil ein entsprechender Methodenapparat für ihre Realisierung fehlt. Nachweislich sind es aber gerade die auf Excellence ausgerichteten Faktoren, die unter den heutigen marktwirtschaftlichen Bedingungen großen Einfluss auf den Geschäftserfolg haben. Dies belegen u. a. Untersuchungen in den USA. [2]

\section{Aufgabenstellung}

Die Teilaufgaben des Forschungsprojektes waren wie folgt zu umreißen:

1. Erarbeitung eines Methodenapparates zur Ermittlung des Handlungsbedarfes von Unternehmen zur angemessenen Erfuillung der Forderungen ihrer interessierten Parteien.

2. Erarbeitung eines Methodenapparates

- zur Identifizierung der wertschöpfenden Kernprozesse und der sie flankierenden Management- und Unterstuitzungsprozesse unter Berücksichtigung der zwischen ihnen anzutreffenden Wechselwirkungen,

- zur Input-/Output-Vernetzung der Geschäftsprozesse auf der Grundlage von möglichst quantitativen Mess- und Zielgrößen sowie

- zur Einbindung der Mess- und Zielgrößen in das Unternehmenscontrolling.

3. Diskussion und Erprobung des Methodenapparates im Sinne seiner wirtschaftlich sinnvollen Einbindung in gelebte QM-Systeme ausgewählter Unternehmen als Beitrag zur Entwicklung umfassenden Qualitätsmanagements auf der Basis der Normenreihe DIN EN ISO 9000:2000 sowie des EFQM-Modells für Excellence. 
4. Verallgemeinerung der Untersuchungsergebnisse und Erarbeitung eines branchenneutralen Leitfadens (TQM-Methodenkatalog) für die Nutzung des entwickelten Methodenapparates zur Gestaltung und Weiterentwicklung betrieblicher QM-Systeme in KMU nach TQM-Aspekten.

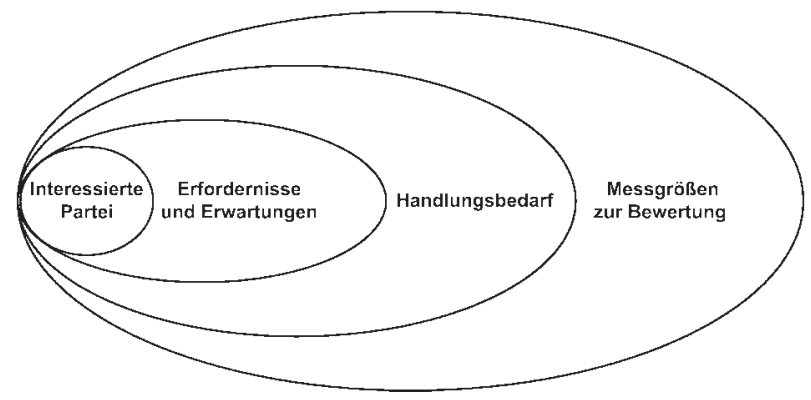

Abb. 1: Ereigniskette zur Ableitung des Handlungsbedarfs und dessen Bewertung aus den Forderungen und Erwartungen interessierter Parteien

\section{Aspekte der DIN EN ISO 9004:2000 und des EFQM-Modells für Excellence}

Als wesentliche Systematisierungsgrundlagen für die Erarbeitung von Lösungsansätzen wurden die Normenreihe DIN EN ISO 9000:2000 sowie das EFQM-Modell fuir Excellence genutzt. Nachfolgende Auszüge aus diesen Systematisierungsgrundlagen unterstreichen die Relevanz des bearbeiteten Forschungsthemas. Sie sollen gleichzeitig als Argumentationshilfe fuir die Nutzung von Ergebnissen des vorliegenden Kataloges dienen.

\section{DIN EN ISO 9000:2000 ff. (Auszïge)}

„Der Erfolg der Organisation hängt vom Verstehen und Zufriedenstellen der gegenwärtigen und zukünftigen Erfordernisse und Erwartungen tatsächlicher und potenzieller Kunden und Endabnehmer sowie dem Verstehen und Berücksichtigen der Erfordernisse und Erwartungen anderer interessierter Parteien ab.“

„Um die Erfordernisse und Erwartungen interessierter Parteien zu verstehen und ihnen zu entsprechen, sollte eine Organisation:

a) ihre interessierten Parteien ermitteln und stets ausgewogen auf deren Erfordernisse und Erwartungen reagieren,

b) erkannte Erfordernisse und Erwartungen in Anforderungen umsetzen,

c) diese Anforderungen auf allen Ebenen der Organisation vermitteln und

d) sich auf Prozessverbesserung konzentrieren, um Wert für die ermittelten interessierten Parteien zu sichern.“ „Die Überwachung der Zufriedenheit der interessierten Parteien erfordert die Beurteilung von Informationen darüber, welche Wahrnehmung bei den interessierten Parteien über die Erfuillung der Anforderungen der interessierten Parteien durch die Organisation herrschen.“ „Die Anwendung eines Systems von Prozessen in einer Organisation, gepaart mit dem Erkennen und den Wechselwirkungen dieser Prozesse sowie deren Management, kann als prozessorientierter Ansatz bezeichnet werden.
Bei der Verwendung in einem QM-System betont ein derartiger Ansatz die Bedeutung:

a) des Verstehens und der Erfuillung von Anforderungen,

b) der Notwendigkeit, Prozesse aus der Sicht der Wertschöpfung zu betrachten,

c) der Erzielung von Ergebnissen bezüglich Prozessleistung und -wirksamkeit und

d) der ständigen Verbesserung von Prozessen auf der Grundlage objektiver Messungen."

"Jeder Prozess stellt eine Abfolge von miteinander in Beziehung stehenden Tätigkeiten bzw. eine Tätigkeit dar, die sowohl Eingaben als auch Ergebnisse hat. Die Leitung sollte erforderliche Ergebnisse von Prozessen sowie die notwendigen Eingaben und Tätigkeiten für die wirksame und effiziente Erlangung dieser Ergebnisse festlegen."

„Die wechselseitige Abhängigkeit von Prozessen kann komplex sein und zu Prozessnetzwerken führen. Um den wirksamen und effizienten Betrieb der Organisation sicherzustellen, sollte die Leitung erkennen, dass das Ergebnis eines Prozesses die Eingabe für einen anderen Prozess oder für mehrere andere Prozesse darstellen kann.“

„Messung ist für das Treffen von auf Fakten beruhenden Entscheidungen von Bedeutung. Die oberste Leitung sollte wirksame und effiziente Messungen, Datenerfassung und -validierung sicherstellen, um die Leistung der Organisation und die Zufriedenheit anderer interessierter Parteien zu gewährleisten.

Beispiele fuir Messung der Leistung einer Organisation schließen ein:

a) Messung und Beurteilung ihrer Produkte,

b) Fähigkeit von Prozessen,

c) Erreichung von Projektzielen und

d) Zufriedenheit der Kunden und anderen interessierten Parteien.“

\section{EFQM-Modell für Excellence (Auszüge)}

\section{Kriterium 1}

Führung, Teilkriterium 1b: Führungskräfte sollen „Sicherstellen, dass ein System für das Management der Prozesse erarbeitet und eingefuihrt wird“.

\section{Kriterium 2}

Politik und Strategie, Teilkriterium 2d: Politik und Strategie werden durch ein Netzwerk von Schlüsselprozessen umgesetzt.

- „Das Netzwerk von Schlüsselprozessen identifizieren und erarbeiten, die benötigt werden, um die Politik und Strategie der Organisation zu realisieren und

- die Effektivität des Netzwerkes der Schluisselprozesse im Hinblick auf die Realisierung von Politik und Strategie überprüifen."

\section{Kriterium 5}

Prozesse, Teilkriterium 5a: Prozesse werden systematisch gestaltet und gemanagt.

- „Die Prozesse in der Organisation einschließlich derjenigen Schlüsselprozesse gestalten, die erforderlich sind, um Politik und Strategie zu realisieren,

- Prozesskennzahlen einfuihren und Leistungsziele festlegen sowie 
- Schnittstellenbelange innerhalb der Organisation und mit externen Partnern lösen, um Prozesse durchgehend effektiv zu managen.“

Diesbezügliche Entwicklungspotenziale bei der Gestaltung und Realisierung von QM-Systemen und TQM-Konzepten legen Analysen von Bewertungen der Bewerberunterlagen zum Ludwig-Erhard-Preis von SCHIMMELPFENNIG [www.deutsche-efqm.de] offen.

Von SCHIMMELPFENNIG benannte Verbesserungspotenziale (abgeleitet aus bewerteten Bewerbern, die keine Vor-Ort-Bewertung erhielten):

- In den Bewerbungsunterlagen finden sich keine durchgängigen Ansätze zur Lösung von Schnittstellenproblemen.

- Es bleibt unklar, wie Prozesse aus der Organisationspolitik und -strategie abgeleitet und priorisiert werden.

- Reviewprozesse zur Effizienz- und Effektivitätsprüifung sind nicht erkennbar oder nicht nachprüfbar in die normalen Geschäftstätigkeiten integriert.

- Messbare Zielvereinbarungen werden vielfach nicht definiert.

- Es werden wenig Kennzahlen zu Prozessleistungen angegeben, der Beitrag von Prozessen zum Geschäftserfolg wird dadurch nicht transparent.

- Es wird nicht dargelegt, wie die Ergebnisse aus Kunden- und Mitarbeiterbefragungen in gezielte Verbesserungsprojekte einfließen.

- Vergleiche mit externen Organisationen werden oft nicht angegeben.

- Es werden nur wenige Trends dargestellt, die eine Entwicklung wichtiger Parameter fuir Mitarbeiter- und Kundenzufriedenheit sowie der Geschäftsergebnisse aufzeigen.

- Nach Auffassung der Autoren [1] verdeutlichen diese Verallgemeinerungen, dass zukünftig dem Problemkreis Prozessorientierung und Geschäftserfolg größeres Augenmerk bei der Realisierung des betrieblichen Qualitätsmanagements einzuräumen ist.

\section{Praxisnahe Lösungsansätze - Beachtung der Spezifika von KMU}

Als Werkzeuge zur Lösungsfindung für den zu erarbeitenden TQM-Methodenkatalog wurden u. a. genutzt:

- Literaturrecherchen,

- Poolbildung zum Forschungsprojekt,

- die Verallgemeinerung vorliegender Erkenntnisse und Erfahrungen bei der systematischen Gestaltung und Realisierung von TQM-Konzepten,

- Prozesskettenanalysen,

- Assessments,

- Workshops mit Experten,

- Führungskräfteworkshops in Erprobungsunternehmen,

- Führungskräfte- und Mitarbeiterschulungen,

- Befragungen interessierter Parteien,

- die Synthese von Lösungsangeboten und deren Erprobung (dazu erfolgte die Bereitstellung der Lösungsentwürfe im Internet mit passwortgeschütztem Zugriff für die Beteiligten am Forschungsprojekt) sowie

- Vorstellung von Lösungsentwürfen in Fachkreisen.
Zur Berücksichtigung der Spezifika von KMU für die Lösungsgestaltung wurden von Beginn der Forschungsarbeiten an folgende Aspekte berïcksichtigt:

- Kompatibilität des Lösungskonzeptes zu den Strategiezielen der weiteren Entwicklung des TQMs in der Region Brandenburg/Berlin (Stärkung/Entwicklung der TQM-Philosophie in KMU, Weiterentwicklung betrieblicher Managementsysteme nach der Normenfamilie DIN EN ISO 9000:2000, Gestaltung integrierter Managementsysteme, Qualitätspreis Berlin-Brandenburg, Qualitätsinitiative Berlin/Brandenburg),

- frühzeitige und möglichst umfassende Einbeziehung von KMU und weiterer Know-how-Träger in die Themenbearbeitung (Erschließung von Know-how sowie Bewertung der Sinnhaftigkeit, Realisierbarkeit, Praxisrelevanz der Lösungen),

- Unterstuitzung der notwendigen Reaktionsfähigkeit und Flexibilität von KMU durch einen durchgängigen und in sich effektiv und flexibel nutzbaren Methodenapparat unter dem Gesichtspunkt einer ganzheitlichen Unternehmensorganisation (z. B. modularer Methodenkatalog, integrierte Managementsysteme, Einbindung in Marketing- und Controllingsysteme der Organisation),

- Berücksichtigung der Branchen- und Unternehmensvielfalt von KMU durch notwendige Allgemeinguiltigkeit der Lösungen (branchenneutraler Methodenkatalog) bei Gewährleistung organisationsspezifischer Adaptionsmöglichkeiten,

- Unterstuitzung der Adaption durch eine möglichst treffende Charakterisierung der Methoden (z. B. Anwendungsbedingungen, Stärken und Grenzen von Methoden), Algorithmierung der Anwendungsschritte (z. B. für die Methodenauswahl), Bereitstellung von Musterdokumenten/-lösungen, Checklisten u. a.,

- Berücksichtigung der begrenzten Ressourcen von KMU durch ein in sich geschlossenes und durchgängiges Methodenkonzept (modularer Methodenapparat), angemessene Werkzeugwahl (Hard-/Software, Methodenkenntnisse, kleine Datenmengen), Angebote für alternative Lösungen (Methodenvielfalt) sowie

- Nutzung geeigneter Formen für die Umsetzung der Forschungsergebnisse (Kommunikationsangebote der TFH Wildau für potenzielle Anwender per Internet, Schulungs-/Weiterbildungsangebote im Rahmen der Qualitätsinitiative Brandenburg).

\section{Einbeziehung von Praxis-Partnern}

Der Gewinnung von Unternehmen für die Mitarbeit in einem Forschungspool lagen Zielstellungen zugrunde wie:

- KMU gehören zu den Branchenfuihrern,

- Präsenz eines hohen Niveaus der betrieblichen Qualitätsmanagementsysteme sowie

- Bereitschaft zur kontinuierlichen Weiterentwicklung des Qualitätsmanagements nach Prinzipien des Total Quality Managements (TQM).

Diese Zielstellungen konnten mit den involvierten Unternehmen in vollem Umfang umgesetzt werden. 
Dem Forschungspool gehörten an:

- Drahtwerk und Stahlhandel Finsterwalde GmbH,

- GfA Gesellschaft für Anlagenbau Guben GmbH,

- Asklepios Klinik Birkenwerder (Qualitätspreisträger Berlin/Brandenburg 2002),

- BRAHMS AG Hennigsdorf(Finalist des Qualitätspreises Berlin/Brandenburg 2002),

- Forster Industrie- und Kesselreinigung GmbH (Finalist des Qualitätspreises Berlin/Brandenburg 2002),

- EuroNorm GmbH, Neuenhagen,

- TÜV Cert GmbH, Berlin,

- TÜV Rheinland/Berlin-Brandenburg und die

- Technische Fachhochschule Wildau, Projektgruppe Qualitätsmanagement.

Formen der Zusammenarbeit waren Arbeitsberatungen, Fuihrungskräfteworkshops im Forschungspool und in einzelnen Unternehmen, Prozessaufnahmen und -bewertungen, Audits sowie die Adaption von Lösungsentwürfen für Unternehmen, deren Erprobung und Integration in die betrieblichen QM-Systeme.

\section{Erarbeitung des TQM-Methodenkataloges}

Der Lösung der Aufgabenstellung für den zu erarbeitenden TQM-Methodenapparat lag das in Abbildung 2 dargestellte Gedankenmodell zu Grunde.

Danach gliederte sich die Lösungsfindung in die Module (Langfassung):

Modul 1 Identifikation interessierter Parteien,

Modul 2 Identifikation der Forderungen und Erwartungen interessierter Parteien,

Modul 3 Forderungsdarstellung,

Modul 4 Identifikation der Angemessenheit der Forderungen und Erwartungen,
Modul 5 Erfassung und Darstellung der durch das Unternehmen umzusetzenden Forderungen und Erwartungen,

Modul 6 Prozessidentifikation, -modellierung und -vernetzung auf der Basis umzusetzender Forderungen und Erwartungen,

Modul 7 Prozessrealisierung und -weiterentwicklung, Modul 8 Messung der Leistungen des Unternehmens im Sinne des Forschungsprojektes,

Modul 9 Managementbewertung.

Die so durchgeführte Lösungsfindung setzte den logischen Ablauf bei der Erschließung der Gesamtaufgabenstellung entsprechend dem Prozessmodell nach der Normenreihe DIN EN ISO 9000:2000 um (vgl. Abb. 3).

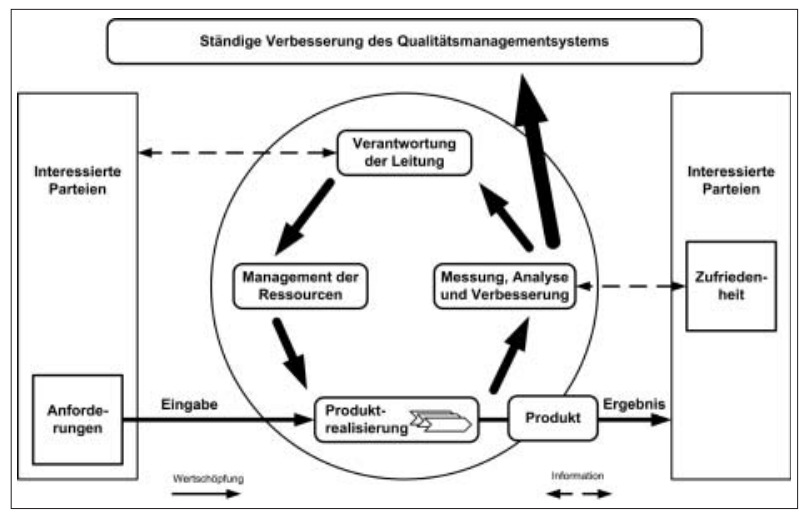

Abb. 3: Prozessmodell nach DIN EN ISO 9004:2000

- Wer sind die interessierten Parteien (Module 1 u. 2)?

- Wie werden die angemessenen Forderungen/Erwartungen umgesetzt (Module 3 bis 7)?

- Welche Ergebnisse werden erreicht (Module 8 u. 9)? Durch die Übernahme dieser Systematik für die Darstellung des Methodenapparates (Kapitel III „Inhaltsbeschreibung nach Modulen“) bietet sie dem Katalognutzer

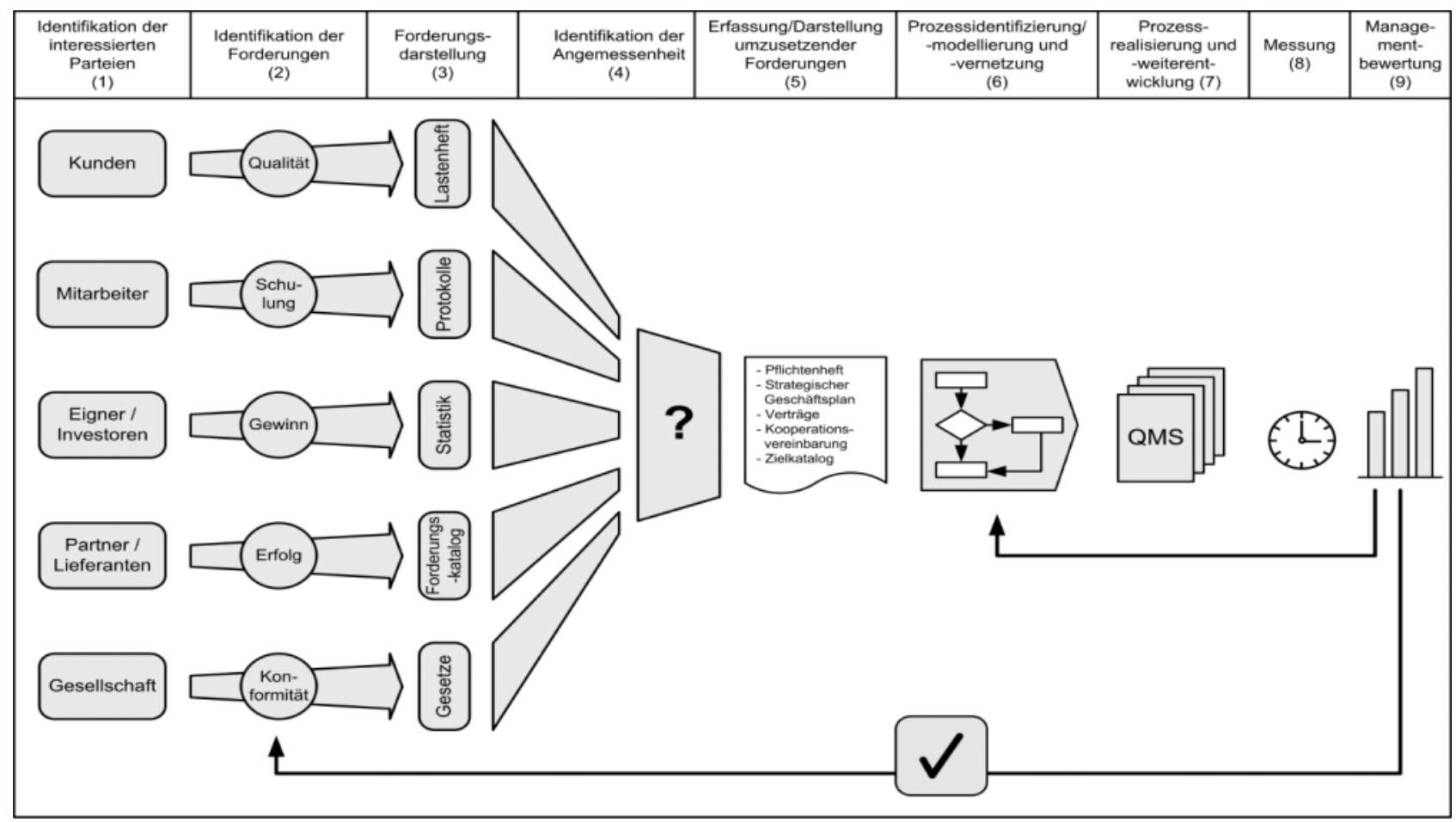

Abb. 2: Lösungs- und Umsetzungsmodell zur Forschungsaufgabe 
einen fachlich begrüindeten und damit einfachen Zugang zu den ihn interessierenden Aufgabenkomplexen (z. B. Prozessvernetzung).

Zur Unterstuitzung der notwendigen Reaktionsfähigkeit und Flexibilität von KMU wurde ein durchgängiger und in sich effektiv und flexibel nutzbarer Methodenapparat (modularer Aufbau) unter dem Gesichtspunkt einer ganzheitlichen Unternehmensorganisation entwickelt.

\section{Unterlagenkonzept des TQM-Methoden- kataloges}

Die Gliederung der Dokumentation des Methodenapparates ist für die Module einheitlich und von ihrer Grundstruktur her der Abbildung 4 zu entnehmen. Die in den Dokumenten vorgeschlagenen Zuständigkeitsregelungen basieren auf dem Referenzorganigramm zum Forschungsprojekt (Abbildung 5). Die strategische Einordnung der Prozesse und ihre Priorisierung erfolgt auf der Basis eines Prozess-Port-Folios (4-Feld-Matrix) entsprechend Abbildung 6.

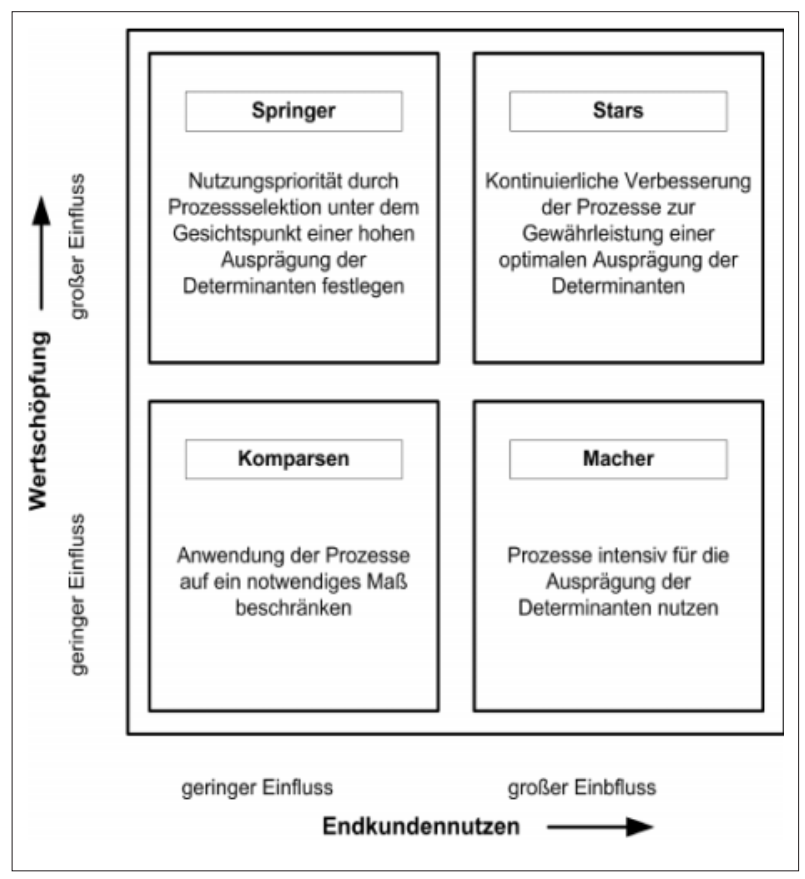

Abb. 6: Vier-Feld-Matrix zur strategischen Einordnung von Prozessen

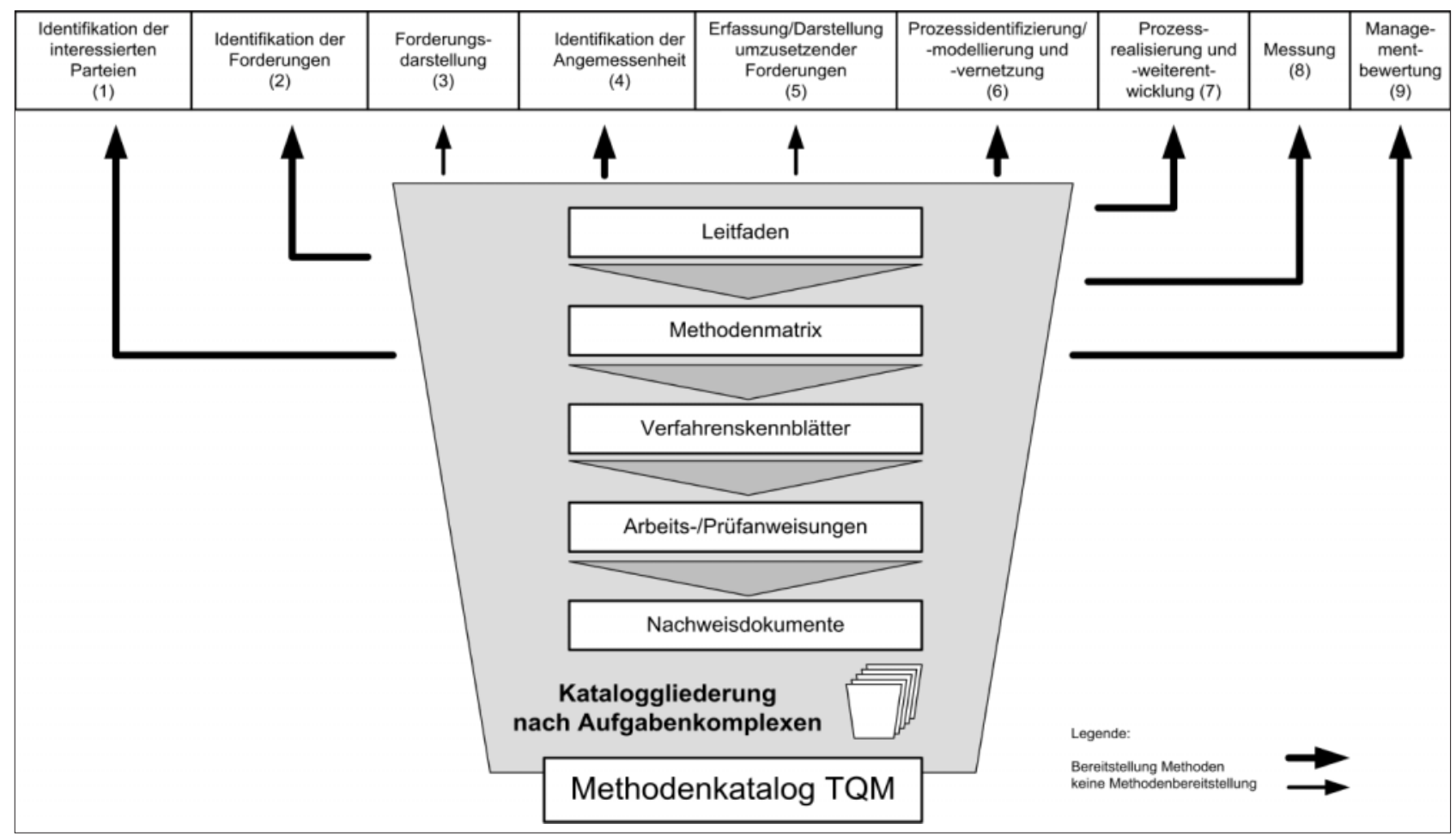

Abb. 4: Struktur des Unterlagenkonzeptes für den Methodenkatalog

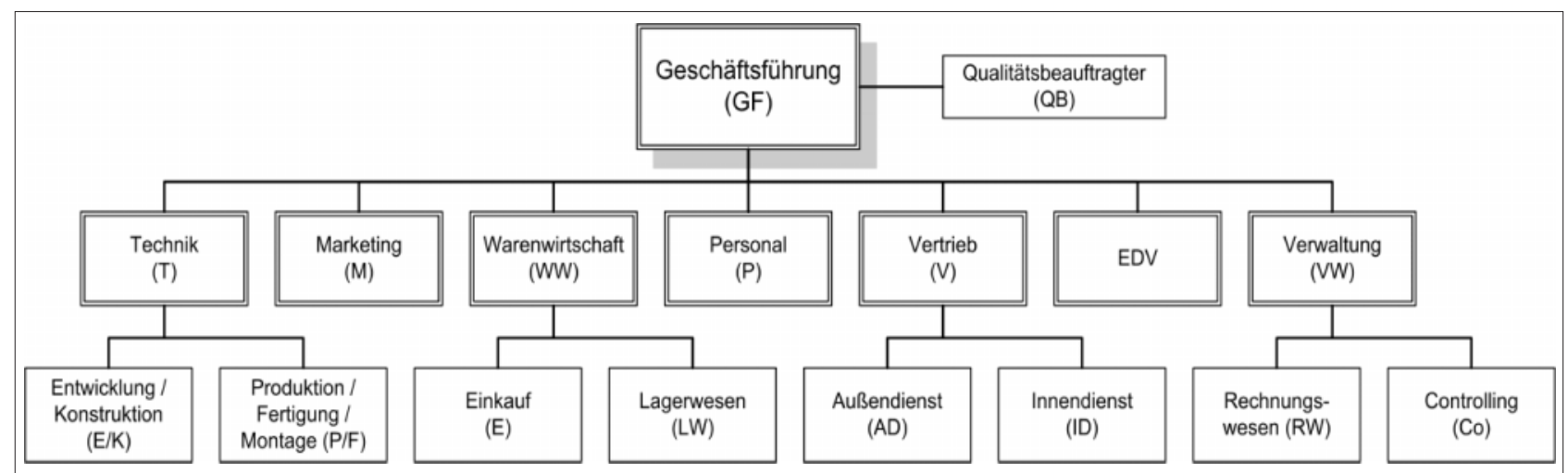

Abb. 5: Referenzorganigramm zum Forschungsprojekt 
In der Tabelle 1 ist die Bewertung der identifizierten Prozesse nach deren strategischen Einordnung dargelegt. Aufbauend darauf lassen sich die Inhalte der Dokumentationsbestandteile des TQM-Methodenkataloges wie folgt charakterisieren:

\section{Leitfaden}

Er beschreibt die prinzipielle Vorgehensweise bei der Bearbeitung einer abgegrenzten Aufgabenstellung entsprechend dem Titel des Moduls. Üblicherweise wird je Modul ein Leitfaden angeboten. Bei einer inhaltlich gebotenen Differenzierung (Modul 6) liegen mehrere Leitfäden zu einem Modul vor. Alle Leitfäden enthalten folgende Gliederungssystematik:

1 Zweck und Geltungsbereich

2 Begriffe

3 Zuständigkeiten

4 Beschreibung des Vorgangs

5 Änderungsdienst

6 Hinweise auf mitgeltende Unterlagen

7 Ablaufalgorithmus

Gegebenenfalls sind den Leitfäden Anlagen zugeordnet.

\section{Methodenmatrix}

In den Methodenmatrizen sind die zum Modul gehörenden Methoden zusammengefasst und bezuiglich ihrer Anwendungsmöglichkeiten charakterisiert. Nachfolgend eine Übersicht zur Methodenauswahl für die Module 1, $2,4,6,7,8$ und 9 .

Methodenauswahl zum Modul 1: Identifikation der interessierten Parteien

- PARETO-Analyse

- Wertschöpfungskettenanalyse

- Internetrecherche zur Ermittlung interessierter Parteien
- Analyse der Gewinn- und Verlustrechnung

- Schriftliche Erhebung

- Publikationsrecherche

Methodenauswahl zum Modul 2: Identifikation von Forderungen und Erwartungen interessierter Parteien

A) Primärerhebungen

- Online-Erhebung

- schriftliche Erhebung

- Mündliche Erhebung

- Beobachtung

B) Sekundärerhebungen

- Unternehmensstatistikanalyse

- Außen-/Kundendienstberichtsanalyse

- Patentrecherche

- Publikationsrecherche

- Fachtagungs- und Messe-/Ausstellungsanalyse

- Internes Audit

- Selbstbewertung

- Benchmarking

- Betriebsvergleich

- Kundenfeedbackanalyse

Methodenauswahl zum Modul 4: Identifikation der Angemessenheit der Forderungen und Erwartungen

- Risikoanalyse

- Fehlermöglichkeits- und Einflussanalyse (FMEA)

- Forderungsbewertung

- Ereignisablaufanalyse

- Publikationsrecherche

- Fähigkeitsanalyse

Methodenauswahl zum Modul 6: Prozessidentifikation, -modellierung und -vernetzung

- Prozessidentifikation und -modellierung

- Ursache-Wirkungs-Diagramm

- Prozesskettenanalyse

- Prioritätsanalyse

\begin{tabular}{|c|c|c|c|c|}
\hline Nr. & Prozessbezeichnung & Prozess & Prozessklasse & Prozesspriorität \\
\hline 1 & Controlling & M5 & Managementprozess & SPRINGER \\
\hline 2 & Einkauf & K4 & Kernprozess & SPRINGER \\
\hline 3 & (Produkt-) Entwicklung & K1 & Kernprozess & STAR \\
\hline 4 & Fehlermanagement & M6 & Managementprozess & KOMPARSE \\
\hline 5 & Fertigung/ Montage & K2 & Kernprozess & STAR \\
\hline 6 & Führungsprozess & M1 & Managementprozess & STAR \\
\hline 7 & Infrastrukturmanagement & $\mathrm{U} 2$ & Unterstiitzungsprozess & KOMPARSE \\
\hline 8 & Interne Audits & M3 & Managementprozess & KOMPARSE \\
\hline 9 & IT-Management & U1 & Unterstïtzungsprozess & SPRINGER \\
\hline 10 & Kontinuierlicher Verbesserungsprozess & M4 & Managementprozess & SPRINGER \\
\hline 11 & Lagerwesen & K7 & Kernprozess & SPRINGER \\
\hline 12 & Leistungsmessung und -überwachung & U6 & Unterstiitzungsprozess & SPRINGER \\
\hline 13 & Lieferantenauswahl und -bewertung & K8 & Kernprozess & KOMPARSE \\
\hline 14 & Managementdokumentation & U3 & Unterstiitzungsprozess & KOMPARSE \\
\hline 15 & Marketing & K3 & Kernprozess & SPRINGER \\
\hline 16 & Personalmanagement & M2 & Managementprozess & SPRINGER \\
\hline 17 & Prozessmanagement & M7 & Managementprozess & STAR \\
\hline 18 & Prüfmittelüberwachung & U5 & Unterstuitzungsprozess & KOMPARSE \\
\hline 19 & Qualitätsberichterstattung & $\mathrm{U} 7$ & Unterstützungsprozess & KOMPARSE \\
\hline 20 & Reklamationsmanagement & K6 & Kernprozess & KOMPARSE \\
\hline 21 & Umwelt- und Arbeitsschutz & M8 & Managementprozess & KOMPARSE \\
\hline 22 & Vertrieb & K5 & Kernprozess & MACHER \\
\hline 23 & Verwaltung & U4 & Managementprozess & KOMPARSE \\
\hline
\end{tabular}

Tab. 1: Prioritätenliste der Prozesse 


\section{- Prozessvernetzung \\ - Benchmarking}

Methodenauswahl zum Modul 7: Prozessrealisierung und -weiterentwicklung

- Qualitäts-Reviews

- Fähigkeitsanalyse

- Prozessverbesserungsmanagement

- Internes Audit

- Selbstbewertung

Methodenauswahl zum Modul 8: Messung der Leistungen des Unternehmens

- Kennzahlenidentifikation und -ermittlung

- Fehleranalyse

- Prozesskettenanalyse
- schriftliche Erhebung
- Selbstbewertung
- Benchmarking
- Betriebsvergleich

Methodenauswahl zum Modul 9: Managementbewertung

- Qualitätscontrolling

- Erfolgsrechnung zur Managementbewertung

- Kundenfeedbackanalyse

- Qualitäts-Reviews

Die Tabelle 2 stellt beispielhaft für das Modul 6 Prozessidentifikation, -modellierung und -vernetzung den Zusammenhang zwischen Methode, Anwendungscharakteristika und dazugehöriges Verfahrenskennblatt dar.

\begin{tabular}{|c|c|c|}
\hline Methode & Anwendungscharakteristika & $\begin{array}{l}\text { dazugehöriges } \\
\text { Verfahrenskennblatt }\end{array}$ \\
\hline $\begin{array}{l}\text { Prozessidentifikation } \\
\text { und -modellierung }\end{array}$ & $\begin{array}{l}\text { - Klassifizierung von Prozessen nach ihrer Stellung im Wertschöpfungs- und Führungsprozess } \\
\text { - Klassifizierung grundsätzlich für Unternehmen aller Größenordnung sowie Branchen und } \\
\text { Profilrichtungen anwendbar zur Schwerpunktsetzung innerhalb der Führungstätigkeit } \\
\text { - Modellierung anwendbar für alle Prozesse } \\
\text { - Modellierung neuer bzw. Weiterentwicklung von Prozessen } \\
\text { - Modellierung als Basis für Prozessanalysen und -optimierung }\end{array}$ & $\begin{array}{l}\text { VK 6.3.1.1 } \\
\text { Prozessidentifikation } \\
\text { und } \\
\text {-modellierung }\end{array}$ \\
\hline $\begin{array}{l}\text { Ursache-Wirkungs- } \\
\text { Diagramm }\end{array}$ & $\begin{array}{l}\text { - systematische Analyse von Prozessen bzw. von Prozessfehlern } \\
\text { - Basis für Prozessweiterentwicklung bzw. -optimierung } \\
\text { - Ermittlung von allen möglichen Ursachen für ein definiertes Problem und Auswahl der Ursachen } \\
\text { mit dem wahrscheinlich größtem Wirkungsanteil } \\
\text { - Anordnung von denkbaren Ursachen und eigentlichem Ereignis/Problem in einem Ursache- } \\
\text { Wirkungs-Diagramm (ISHIKAWA-Diagramm) }\end{array}$ & $\begin{array}{l}\text { VK 6.3.1.2 } \\
\text { Ursache-Wirkungs- } \\
\text { Diagramm }\end{array}$ \\
\hline Prozesskettenanalyse & $\begin{array}{l}\text { - Analyse nach verschiedenen Zielkriterien, z. B. Durchlaufzeit- oder Kostenminimierung } \\
\text { - Rechnerunterstüitzte Bearbeitung möglich und Auswahl von Vorzugsvarianten beim Vorliegen } \\
\text { verschiedener Gestaltungsentwürfe für Prozesse } \\
\text { - Anwendung für Prozessentwicklung, z. B. aufgrund neuer bzw. veränderter Kundenforderungen } \\
\text { - Verfahren zur Bewertung der Leistungsfähigkeit, Wirtschaftlichkeit Fehlerfreiheit von Prozessen } \\
\text { - Bewertung und Einordnung der Analysenergebnisse setzt gewöhnlich das Vorliegen von } \\
\text { Zielgrößen/Benchmarks (z. B. für die Zielkriterien) }\end{array}$ & $\begin{array}{l}\text { VK 6.3.1.3 } \\
\text { Prozess- } \\
\text { kettenanalyse }\end{array}$ \\
\hline Prioritätsanalyse & $\begin{array}{l}\text { - für alle Geschäftsprozesse nutzbar, } \\
\text { - priorisiert Geschäftsprozesse, z. B. nach betriebsspezifischen Zieldeterminanten wie } \\
\text { Kundennutzen oder Wertschöpfung, } \\
\text { - Festlegen zur Schwerpunktsetzung im Prozessmanagement }\end{array}$ & $\begin{array}{l}\text { VK 6.3.1.4 } \\
\text { Prioritätsanalyse }\end{array}$ \\
\hline Prozessvernetzung & $\begin{array}{l}\text { - Ermittlung der Wechselwirkungen zwischen Prozessen im Rahmen der internen } \\
\text { Wertschöpfungskette einer Organisation } \\
\text { - Input-/Outputermittlung und deren Beschreibung } \\
\text { - Output-/Inputvernetzung der Geschäftsprozesse im Sinne der Gewährleistung einer hohen } \\
\text { Wirksamkeit und Effizienz des Qualitätsmanagements einer Organisation } \\
\text { - anspruchsvolle Aufgabenstellung, deren Lösung in Abhängigkeit vom Reifegrad des QM-Systems } \\
\text { einer Organisation ggf. schrittweise zu realisieren ist }\end{array}$ & $\begin{array}{l}\text { VK 6.3.1.5 } \\
\text { Prozessvernetzung }\end{array}$ \\
\hline Benchmarking & $\begin{array}{l}\text { - für die problemspezifische Verdichtung und Analyse von vorhandenen Daten z. B. Branchen- } \\
\text { daten, Konkurrenzdaten, allgemeine Daten zur regionalen und überregionalen Wirtschaftslage } \\
\text { - zur Ermittlung von Trends } \\
\text { - zur Zielidentifikation bzw. zur Prüfung der Zielrealisierung } \\
\text { - zur Bewertung verallgemeinerungsfähiger, komplexer Zusammenhänge } \\
\text { - bei nationalem und internationalem Informationsbedarf } \\
\text { - zur systematischen Ermittlung von Abweichungen, Schwachstellen/Problemen bezogen auf } \\
\text { Produkte, Dienstleistungen, Prozesse } \\
\text { - für den Vergleich mit den „Besten“ } \\
\text { - bei operativen Informationsbedarf } \\
\text { - zur Ermittlung von Stand der Wissenschaft } \\
\text { - zur Anwendung für die Prozessentwicklung, z. B. aufgrund von Zielgrößen/Benchmarks } \\
\text { (bezieht sich auf Modul 6) } \\
\text { - zur Bewertung von Prozessen und Ermittlung von Verbesserungspotentialen (bezieht sich auf } \\
\text { Modul 6) } \\
\text { - zur Ermittlung von kritischen Erfolgsfaktoren und Zieldeterminanten (bezieht sich auf Modul 6) } \\
\text { - zur Bewertung der Leistungen des Unternehmens auf der Basis von Zielgrößen/Benchmarks } \\
\text { (bezieht sich auf Modul 8) }\end{array}$ & $\begin{array}{l}\text { VK 2.3.2.8 } \\
\text { Benchmarking }\end{array}$ \\
\hline
\end{tabular}

Tab. 2: Methodenmatrix Prozessidentifikation, -modellierung und -vernetzung 


\section{Verfahrenskennblätter}

Für jede in den Methodenmatrizen dargestellte Methode liegt im Katalog ein eigenes Verfahrenskennblatt vor. In ihnen wird der Vorgang für die Methodenanwendung beschrieben. Alle Verfahrenskennblätter enthalten folgende Gliederungssystematik:

1 Anwendungscharakteristika

2 Anwendungen und Periodizitäten

3 Beschreibung des Vorgangs

4 Voraussetzungen

5 Hinweise auf geltende Unterlagen

6 Ablaufalgorithmus

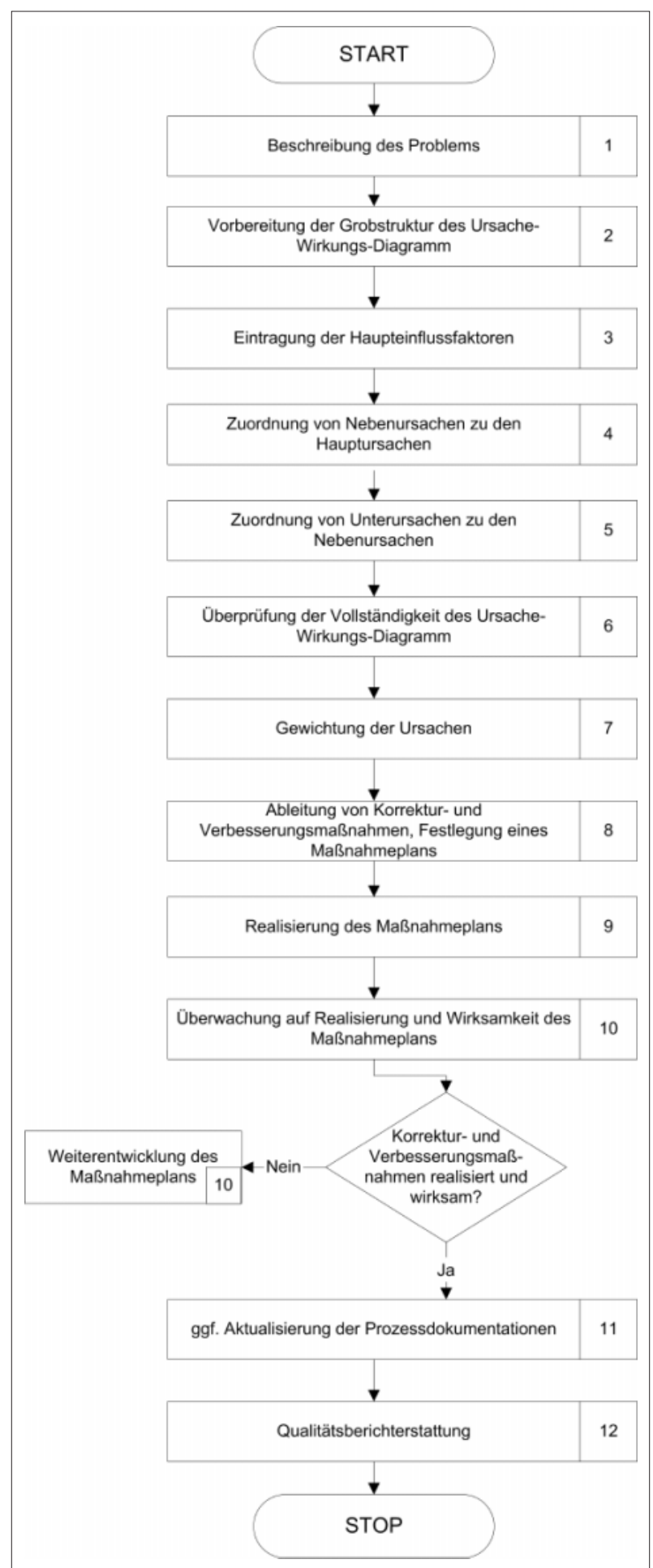

Abb. 7: Ablaufalgorithmus des Ursache-Wirkungs-Diagramms Anwendung zur Prozessidentifikation, -modellierung und -vernetzung
Den Verfahrenskennblättern sind uiblicherweise Arbeitsund Prüfanweisungen und/oder Nachweisdokumente zugeordnet. Auf Grund der Multivalenz verschiedener Methoden wurden einzelne Methoden im Katalog verschiedenen Modulen zu geordnet (z. B. ist Verfahrenskennblatt VK 2.3.2.2 „Schriftliche Erhebung“ auch Bestandteil der Module 1, 2 und 8). Bei der Darstellung der Prozesse und ihrer Vernetzungen wird zum einen von dem jeweils anzustrebenden Niveau (Reifegrad) der Prozessübersicht, zum anderen von der dazugehörigen Prozessvernetzung ausgegangen (s. a. Abbildung 8).

\section{Niveaus (Reifegrade) der Prozessübersicht}

\begin{tabular}{|l|l|}
\hline Reifegrad & Umfang der Dokumentation \\
\hline Ia & Benennung Prozess/Bezugsprozess \\
\hline Ib & $\begin{array}{l}\text { Ia + Ausweis der Art des Prozesses, wie Kern-, } \\
\text { Management- oder Unterstiitzungsprozess }\end{array}$ \\
\hline II & $\begin{array}{l}\text { Ia + Ib + Ausweis der In- und Outputs sowie } \\
\text { Darstellung der Schnittstellen. }\end{array}$ \\
\hline III & $\begin{array}{l}\text { Ia + Ib + II + Vervollständigung der Schnittstellen } \\
\text { (Vernetzung) }\end{array}$ \\
\hline
\end{tabular}

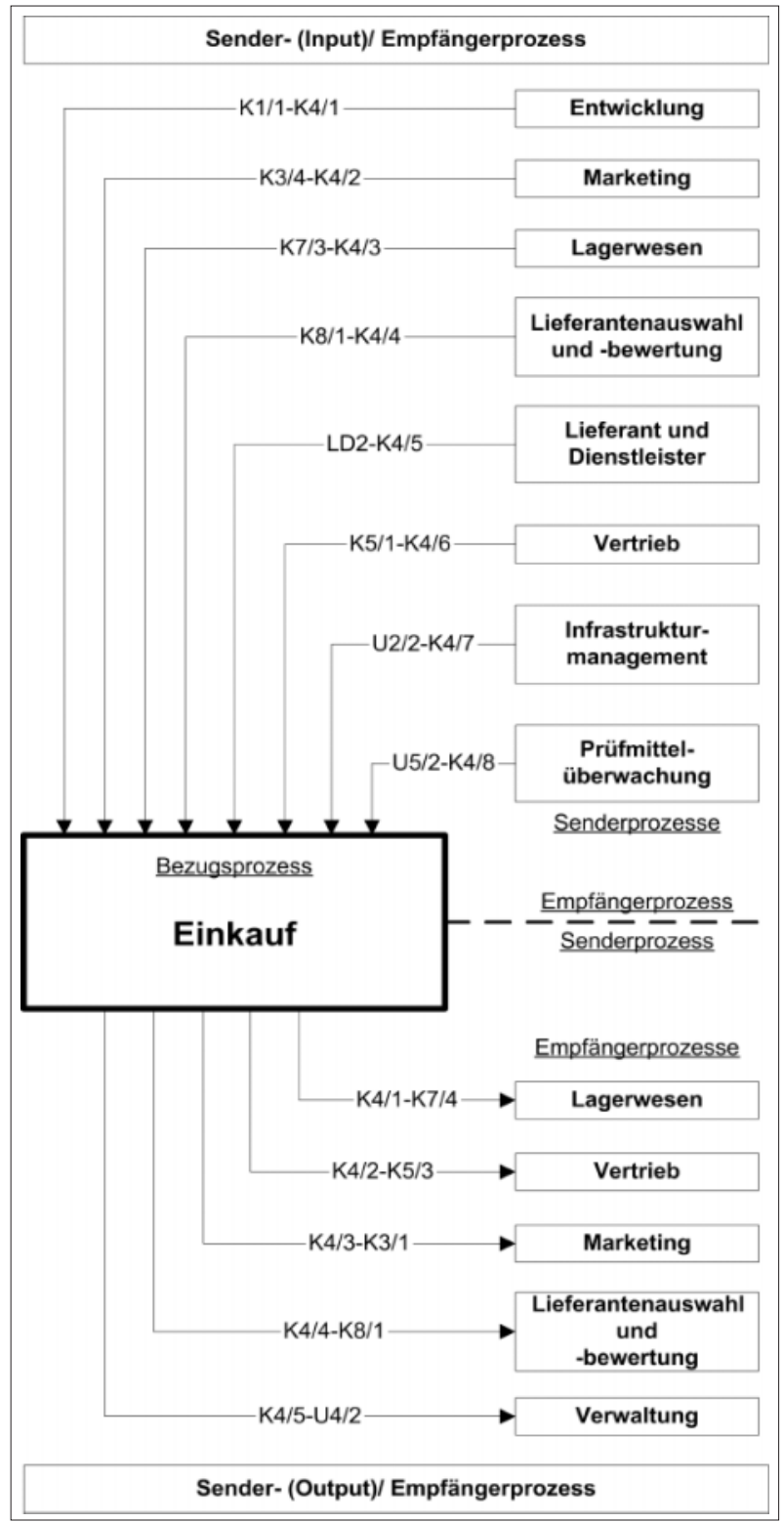

Abb. 8: Beispiel für die vollständige Kennzeichnung Schnittstellen eines Prozesses (Beispiel Einkauf) 
Niveaustufen der Prozessvernetzung

\begin{tabular}{|l|l|l|}
\hline $\begin{array}{l}\text { Niveau- } \\
\text { stufe }\end{array}$ & Darstellung & Anwendungsfeld \\
\hline A & Prozessuibersicht - Reifegrad III & $\begin{array}{l}\text { Führungsinstrument } \\
\text { des Managements }\end{array}$ \\
\hline B & $\begin{array}{l}\text { Niveaustufe A + Verankerung der } \\
\text { Vernetzungen in Anweisungsdoku- } \\
\text { menten, z. B. in Verfahrens- oder } \\
\text { Arbeitsanweisungen }\end{array}$ & $\begin{array}{l}\text { wie Niveaustufe A + } \\
\text { Prozesseigner }\end{array}$ \\
\hline C & $\begin{array}{l}\text { Niveaustufe B + graphische Dar- } \\
\text { stellung der Prozessvernetzung } \\
\text { (im Weiteren „Prozessvernetzung“) }\end{array}$ & $\begin{array}{l}\text { umfassende Nutzung } \\
\text { im Sinne „Exzellenz“ }\end{array}$ \\
\hline
\end{tabular}

\section{Arbeits- und Prüfanweisungen}

Sie beschreiben den Vorgang und die Rahmenbedingungen für die Realisierung einer Aufgabenstellung, z. B. für die Identifizierung, Ermittlung und Bewertung einer Kennzahl.

\section{Nachweisdokumente}

Zu den Nachweisdokumenten gehören Formulare, Musterdokumente und erklärende Beispiele zur Anwendung der jeweiligen Methode.

Eine Sonderheit stellen die Module 3 und 5 dar. Da es sich bei diesen Modulen vom Inhalt her nur um die Erfassung/Zusammenstellung von Informationen handelt, werden für sie keine Methoden vorgeschlagen (s. Abb. 6). Es erfolgt nur eine stabstrichartige Aufzählung relevanter Erfassungs-/Darstellungsformen. Dabei wird davon ausgegangen, dass gerade diese Aspekten in betrieblichen QM-Systemen umfassend geregelt werden.

Der vollständige TQM-Methodenkatalog weist die nachfolgende Gliederung auf:

Kapitel I Einführung

Kapitel II Nutzungsempfehlungen

Kapitel III Inhaltsbeschreibung nach Modulen

- Modul 1 Identifikation der interessierten Parteien

- Modul 2 Identifikation von Forderungen und Erwartungen interessierter Parteien

- Modul 3 Forderungsdarstellung

- Modul 4 Identifikation der Angemessenheit der Forderungen und Erwartungen

- Modul 5 Erfassung und Darstellung der durch das Unternehmen umzusetzenden Forderungen und Erwartungen

- Modul 6 Prozessidentifikation, -modellierung und -vernetzung auf der Basis umzusetzen der Forderungen und Erwartungen

- Modul 7 Prozessrealisierung und -weiterentwicklung

- Modul 8 Messung der Leistungen des Unternehmens im Sinne des Forschungsprojektes

- Modul 9 Managementbewertung

Kapitel IV Zusammenfassung

Kapitel V Beispielunterlagen

Kapitel IV Ergebnisse aus der Erprobung u. Protokolle

\section{Nutzung des TQM-Methodenkataloges}

Die Ansprïche an Methodenkenntnisse bei der Nutzung des Kataloges sind differenziert. Insbesondere unter Berücksichtigung begrenzter Ressourcen in KMU wurden die Werkzeugauswahl und die Form der Methodenbeschreibung so gewählt, dass üblicherweise keine oder nur geringe Methodenkenntnisse erforderlich sind. Wenn sinnvoll, wurden alternative Lösungsangebote bereitgestellt. Die Nutzung des vorliegenden TQM-Methodenkataloges ist in Abbildung 9 beispielhaft dargestellt.

Inhaltlich gehen die Beschreibungen (z. B. in Verfahrenskennblättern) teilweise wesentlich über die Aufgabenstellung hinaus. Als ein Beispiel kann dafür das Verfahrenskennblatt VK 4.3.1.1 „Risikoanalyse“ genannt werden. Damit wurde das Ziel verfolgt, dem Katalognutzer komplexe und gleichzeitig in sich geschlossene Lösungsansätze anbieten zu können. Die gewählte Form der Methodendarstellung und die beigefuigten Beispiele sollen eine betriebliche Nutzung des Kataloges in KMU erleichtern. Neben einem durchgängigen Beispiel zur Prozessidentifikation, -modellierung und -vernetzung, zusammengestellt aus anonymisierten Ergebnissen der Erprobung (Kapitel V „Beispielunterlagen“), enthält der Katalog eine große Anzahl von Lösungsbeispielen. Nachfolgende Aufzählung vermittelt einen Überblick zu diesen Einzelbeispielen:

AP 2.4.2.1.1.........Ermittlung der Kennzahl StammkundenUmsatzanteil

AP 2.4.2.9.1..........Ermittlung der Personalproduktivität (PP)

ND 1.5.1.1.1 ........PARETO-Analyse am Beispiel Ermittlung der Kundenstruktur

ND 2.5.1.2.4 ........ Mitarbeiterfragebogen zur Identifikation interessierter Parteien

ND 2.5.1.2.1 ........Kundenfragebogen (Identifikation Kundenforderungen)

ND 2.5.1.3.1 ........ Interviewleitfaden für Lieferanten

ND 2.5.1.4.1 ........ Telefoninterviewleitfaden

ND 2.5.2.5.1 ........Checkliste zur Ermittlung von Erwartungen und Forderungen der Kunden

ND 2.5.2.1.1 ........Scorecard Stammkunden-Umsatzanteil

ND 2.5.2.10.1 .....Fehlerkatalog

ND 2.5.2.2.2 .......Erhebungsbogen Außendienst

ND 2.5.2.4.1 .......Publikationsrechercheplan

ND 2.5.2.4.2 ........Ergebnisbericht der Publikationsrecherche

ND 2.5.2.5.1 ........Messebesucherbewertung

ND 2.5.2.5.2 ........ Erhebungsbogen für Messestandbesucher

ND 2.5.2.5.3 ........Checkliste Wettbewerberanalyse auf Messen

ND 2.5.2.7.4 ........Checkliste für die Selbstbewertung und Ergebniserfassung

ND 6.5.1.2.1 ........Ursache-Wirkungs-Diagramm am Beispiel Transportschäden an ausgelieferten Warensendungen

ND 6.5.1.3.1 ........Schwachstellenanalyse nach Varianten

ND 2.5.1.2.2 ........ Kundenfragebogen (Identifikation Kundenzufriedenheit, Ermittlung Kundenzufriedenheitsindex) 
ND 2.5.1.2.3 ........Mitarbeiterfragebogen (Identifikation Mitarbeiterzufriedenheit, Ermittlung Mitarbeiterfriedenheitsindex)

ND 8.5.1.1.3 ........Performance Cockpit

ND 8.5.1.2.1 ........Fehlerkatalog (hier am Beispiel Motorenfertigung/-instandsetzung)

Die Dokumente des TQM-Methodenkataloges wie Leitfäden, Methodenmatrizen, Verfahrenskennblätter, Arbeits- und Prüfanweisungen, Nachweisdokumente sowie die Beispielunterlagen sind mit Microsoft-OfficeSoftware (Word, Visio, Excel usw. (Version 97)) erstellt. Insgesamt enthält der TQM-Methodenkatalog über 100 Musterdokumente und ausgewählte Beispielunterlagen. Die CD-ROM zum TQM-Methodenkatalog enthält zur besseren Navigation eine PDF-Datei sowie zusätzlich die jeweiligen Office-Dokumente, die entsprechend den betrieblichen Bedingungen durch die Nutzer angepasst und erweitert werden können.

\section{Zusammenfassung}

Der TQM-Methodenkatalog unterstiitzt die Erschließung von Schwerpunktaspekten bei der Weiterentwicklung des betrieblichen Qualitätsmanagements im Sinne der Identifikation von Forderungen und Erwartungen interessierter Parteien eines Unternehmens und deren angemessene und wirksame sowie effiziente Umsetzung durch eine durchgängige Prozessgestaltung auf der Basis input-/ outputvernetzter Geschäftsprozesse (Schnittstellenproblematik) als Voraussetzung für die Gewährleistung einer hohen Zufriedenheit der interessierten Parteien und eines nachhaltigen Geschäftserfolges. Er setzt damit direkt die Charakteristika des Prozessmodells nach DIN EN ISO 9004:2000 um.

\section{Anmerkungen/Literatur}

[1] Stegemann, Gerd; Petschick, Nicole; Wohlfahrt, Ingolf; Kauschke, Steffen u. a.: TQM-Methodenkatalog zur Input-/ Outputvernetzung und Bewertung von Geschäftsprozessen in KMU, Technische Fachhochschule Wildau, Projektgruppe Qualitätsmanagement, 2003, unveröffentlicht.

[2] Singhal, Hendricks, Schnauber; QZ 45 (2000) 12, S. 1537 ff.

\section{Autor}

Dr.-Ing. Ingolf Wohlfahrt

Technische Fachhochschule Wildau

Fachbereich Ingenieurwesen/Wirtschaftsingenieurwesen

Projektgruppe Qualitätsmanagement

Tel. +493375 508-913

E-Mail: qmwohlfa@igw.tfh-wildau.de

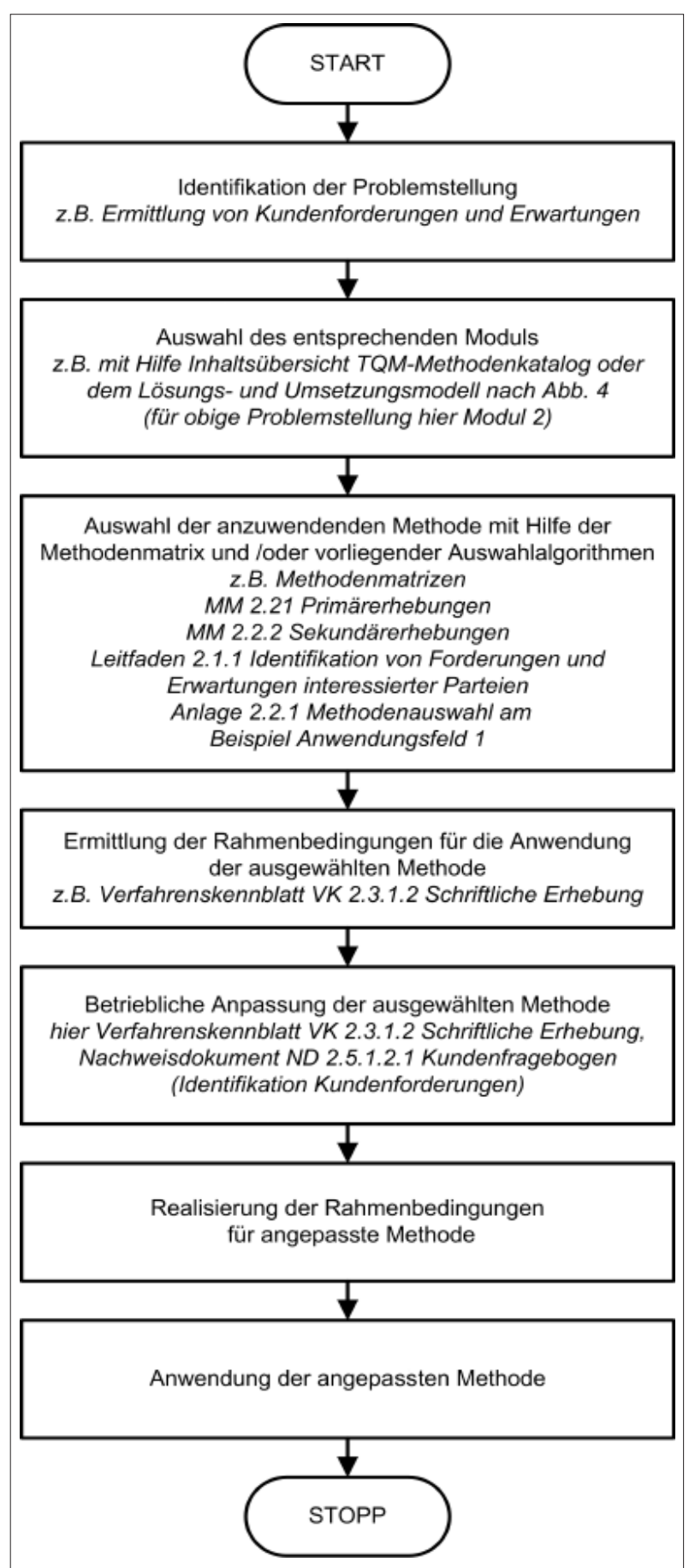

Abb. 9: Prinzipielle Vorgehensweise bei der Nutzung des TQM-Methodenkatalogs 